\title{
Control of a fast switching valve for digital hydraulics
}

\author{
N. P. Sell , D. N. Johnston , A. R. Plummer, and S. Kudzma \\ Dept. of Mechanical Engineering, University of Bath, Bath, UK \\ E-mail: N.P.Sell@bath.ac.uk
}

\begin{abstract}
The success of digital hydraulics is dependent on the ability to produce digital hydraulic valves that can supply both high flows and high switching rates. This paper will elucidate the control scheme of, and results obtained from, such a valve which has been developed at the University of Bath. It will be shown that through the combination of iterative learning control and state variable feedback control it is possible to accurately follow a $100 \mathrm{~Hz}$ PWM signal on a valve designed for high flow rates $(651 / \mathrm{min}$ at $10 \mathrm{bar})$, making it well suited to use in a Switched Reactance Hydraulic Transformer.
\end{abstract}

Keywords: Digital Hydraulics, Learning Control, System Identification, Complimentary filters.

\section{Introduction}

The field of digital hydraulics is seen as pivotal to the ongoing success and applicability of fluid power as a whole, as it can offer significantly more efficient hydraulic circuit designs in many applications. The realisation of these savings is, however, currently limited by hardware and more specifically the fast switching valves required.

This reliance on high speed switching valves has resulted in numerous papers on the subject, these take one of two routes. The first is looking into using commercially available valves. This approach was shown to be successful, though not ideal in many cases, for example controlling a three camber cylinder [1], for use in a digital hydraulic transformer [2] and the creation of a higher flow rate switching valve by modifying existing 4/2 directional valves [3]. The second route is to develop valves specifically for use in digital hydraulics. Many novel designs have arisen to meet the contrary demands of high flow and fast switching, these have included a bistable hammer design [4], a small needle valve [5] and a rotating valve [6]. There also exists a commercial solution [7]. All of these valves all have different applications in mind and as such have a range of switching frequencies, flow rates and package sizes.

There is however, currently, no valve that combines the high flow rate and switching speeds needed for us in a Switched Reactance Hydraulic Transformer (SRHT). The Centre for Power Transmission and Motion Control at the University of Bath is currently developing such a valve. A SRHT works on a similar premise to the buck converter in power electronics, as can be seen in Figure 1, though frequently a buck converter utilises a diode in the ground connection in place of the switch.

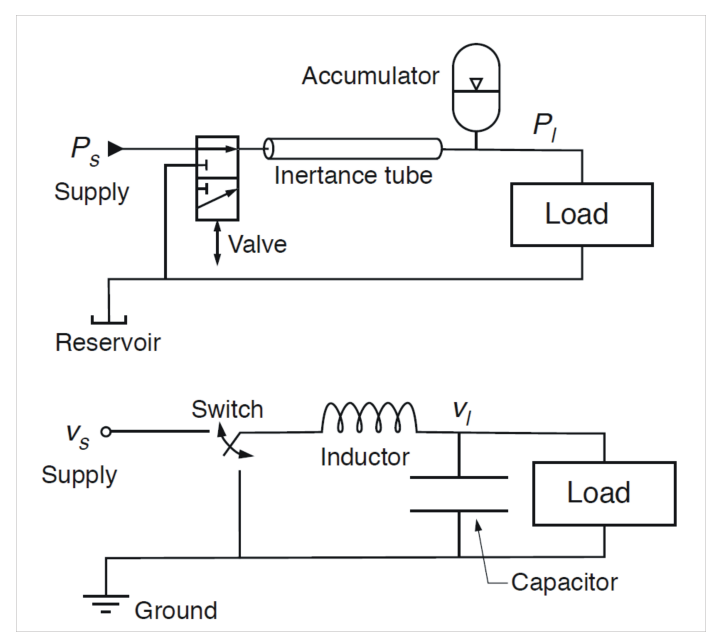

Figure 1: Comparison between SRHT and buck converter

By switching between low pressure and high pressure sources quickly it is possible to generate a range of different pressure and flow rates without the need for throttling and the associated losses. In order for the SRHT to work the fast switching valve must be able to track pulse width modulated (PWM) signals of at least $100 \mathrm{~Hz}$ [8]. A brief overview of the valve's design, simulation and modelling will be given below, before describing the development of the control scheme used with the valve. Finally experimental results showing the valve's ability to reproduce a $100 \mathrm{~Hz}$ square wave will be presented.

\section{Valve Design}

In order to meet the high speed requirements of an SRHT, whilst maintaining the high flow rate $(65 \mathrm{~L} / \mathrm{min}$ at $10 \mathrm{bar}$ pressure drop) needed to make the SRHT practical, a novel three 
stage design has been adopted. The primary stage is a Moog high speed servo valve which is used to direct high pressure flow (up to 200bar) into an equal area actuator. This is directly attached to the main spool with its multiple grooves. These multiple grooves have the advantage of exposing a large flow area, $37.7 \mathrm{~mm}^{2}$, for a small spool displacement, $0.1 \mathrm{~mm}$. It is this high flow gain that makes the valve suited to digital hydraulics, giving both a high frequency of switching and a high flow rate. Due to the nature of the primary stage it is also possible to operate the valve as a simple proportional control valve if this proves more efficient in a specific circumstance. To aid with the control of the valve an accelerometer and position sensor are also incorporated into the valve housing, the former at the top and the latter the bottom of the valve as shown in Figure 2 below.

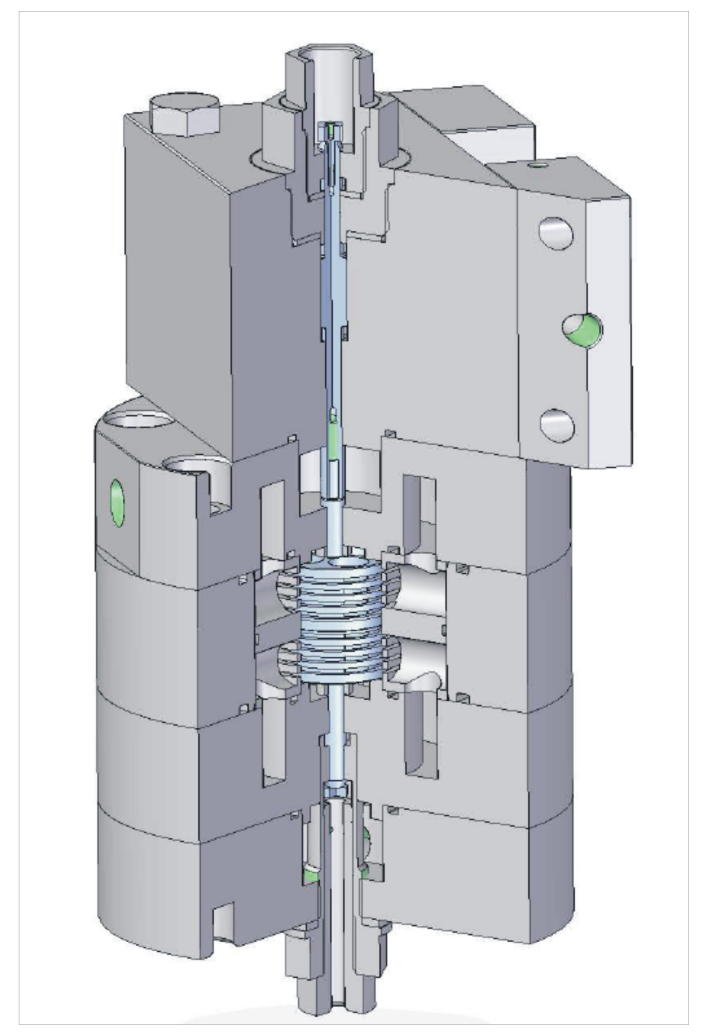

Figure 2: CAD model of the fast switching linear valve

More detailed explanation of the valve's design and construction can be found in a previous paper [9].

\section{Experimental Set-up}

In order to validate the valves design and control strategy the test rig shown in Figure 3 was created. It uses two power packs, one to supply flow to the main stage and the other to supply high pressure flow to the pilot stage. Currently the pressures and flow rates in the main stage are not being controlled but instead are being used to provide lubrication, therefore the pressure difference between the two ports is currently not in excess of 10bar and the high pressure not above 40bar.

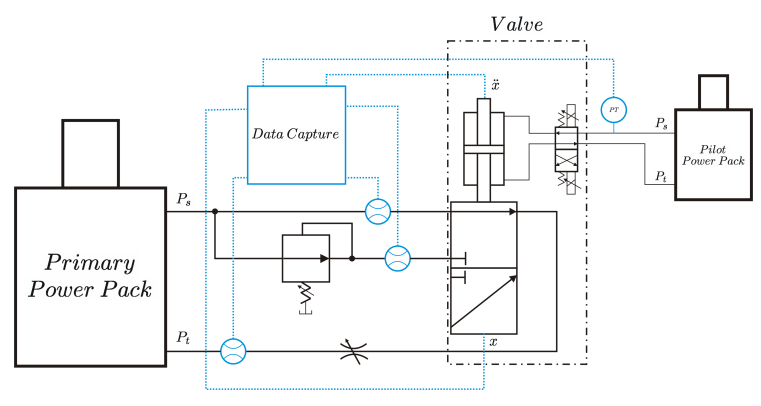

Figure 3: Experimental Set-up

The data provided by the pressure transducers and flow meters is currently used for monitoring purposes only, whilst the Keyence EX-110 position sensor and Kistler 8730AE500 accelerometer housed within the valve body are used for control. Both of these sensors are band limited with the position sensor accurate between $0-400 \mathrm{~Hz}$ and the accelerometer between $20-2 \mathrm{kHz}$. It is desirable to have a larger bandwidth for the position measurements to enable faster and more accurate control. This is achieved by low pass filtering the position data and then combining it with high pass filtered acceleration data which has been integrated twice. The high and low passed signals are simply added together, this can be done as the two filter designs were made complementary [10]. Complementary filters are filters whose sum in the frequency domain is unity at all points. The magnitude of the two filters is shown in Figure 4.

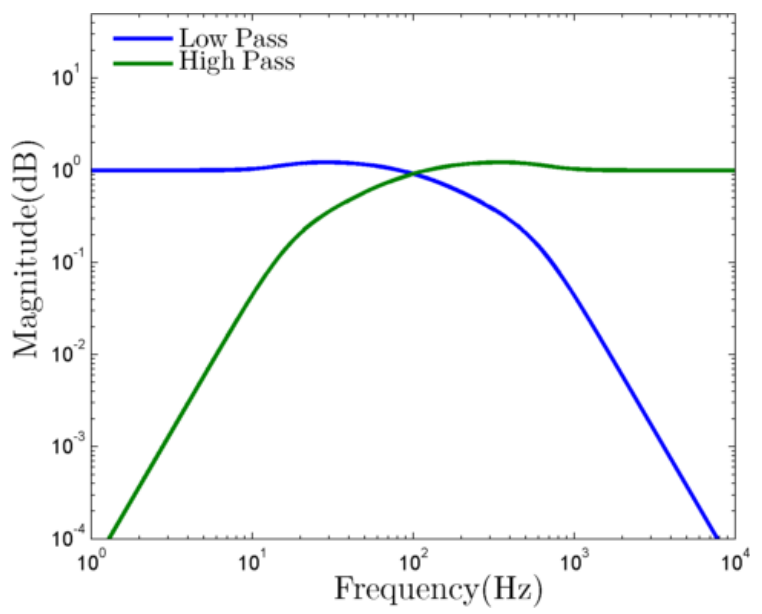

Figure 4: Magnitude of complementary filters

Therefore at the point of crossover $(100 \mathrm{~Hz}$ in this case) the signal is an equal combination of the two sources, at frequencies below this point the position signal makes a greater contribution. A similar technique can be used to improve the bandwidth of the acceleration data and also to provide high bandwidth and accurate velocity measurements. It is necessary to differentiate the position data in both of these cases which is achieved by increasing the order of the numerator in the low pass filter. 


\section{Simulation and Modelling}

The first stage in the development of the spool position control system was to create a mathematical model of the valve for use in simulation. Figure 5 shows a block diagram of the model developed.

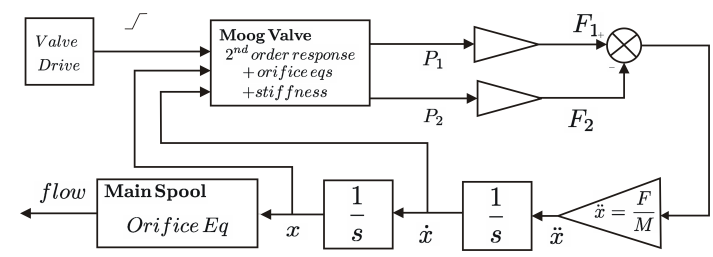

Figure 5: Block diagram of valve model

\subsection{Model Validation}

In order to validate the valve model an empirically tuned PID controller was used to stabilise the valve and provide some data for comparison purposes. A comparison between the simulated and measured results can be seen in Figures 6 and 7.

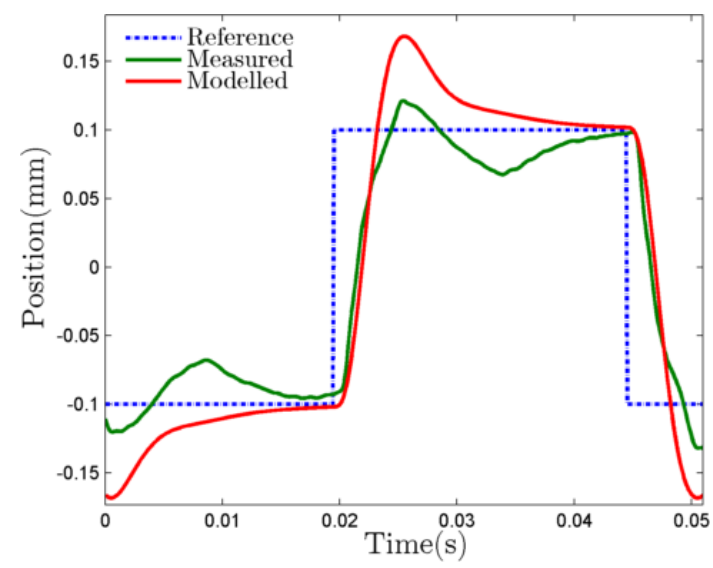

Figure 6: Comparison of simulated and actual response under PID control at $20 \mathrm{~Hz}$

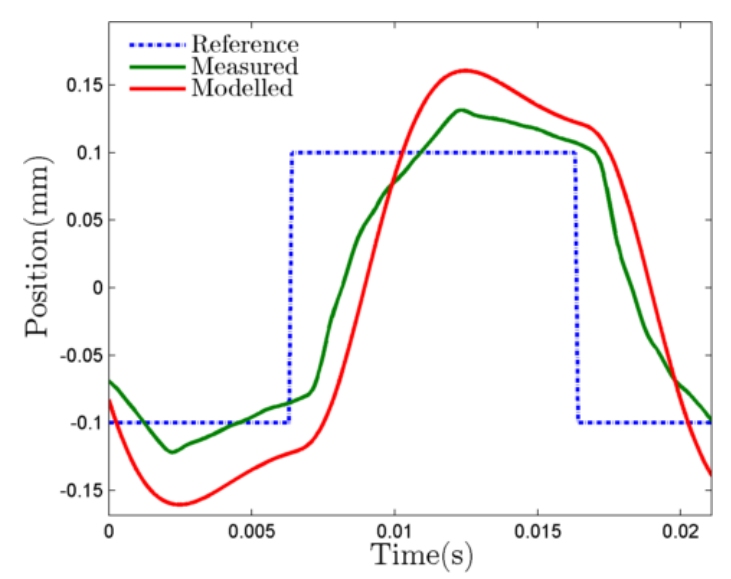

Figure 7: Comparison of simulated and actual response under PID control at $50 \mathrm{~Hz}$
The mismatch between the simulated and recorded response is believed to be mainly the result of leakage between the lower chamber of the second stage and the main stage. Therefore system identification methods were used to estimate an improved linear model of the valve, the step response of this new model can be seen in Figure 8 along with the response of the originally derived system.

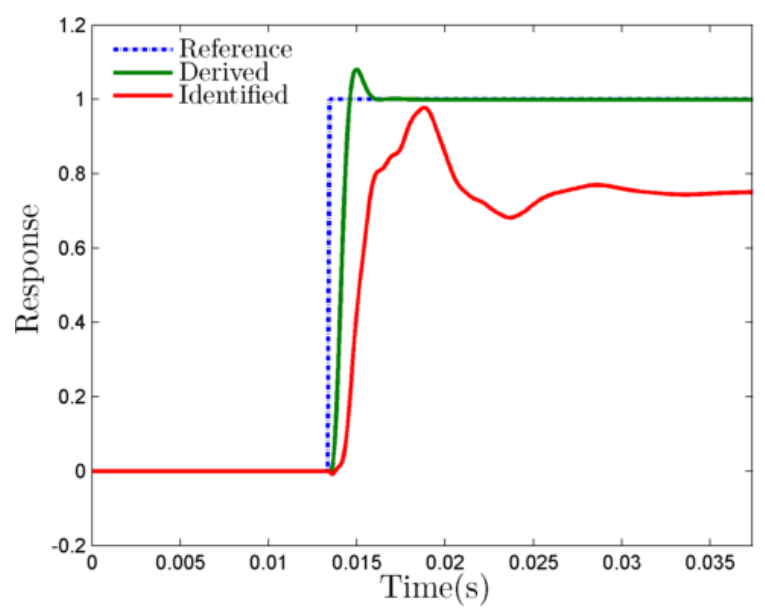

Figure 8: Comparison of empirical and identified models step response

\section{State Variable Feedback Control}

Whilst it is apparent that a classic PID controller could be used with the valves there are two characteristics of the statespace model that make the use of State Variable Feedback (SVF) appealing. Firstly high bandwidth and accurate data for the valves position, velocity and acceleration is available courtesy of the complementary filter network outlined in section 3, meaning that the system if fully observable. Secondly it is possible to prove that the state space model is fully controllable as the controllability matrix is of full rank [11]. This combination of observability and controllability mean that it is possible to move all the open-loop poles to any arbitrary closed-loop location using SVF [12]. The block diagram of the SVF system can be seen in Figure 9.

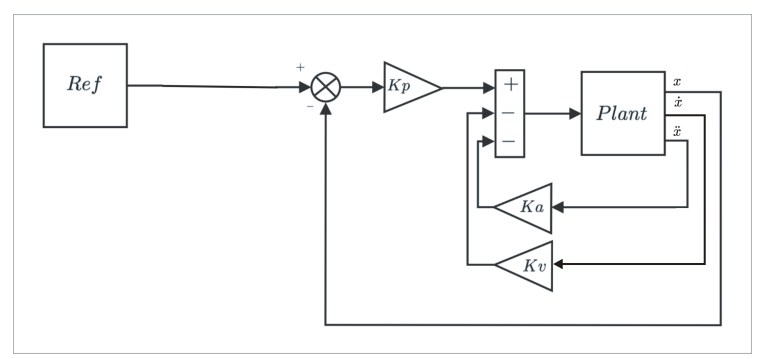

Figure 9: SVF system block diagram

Ackerman's method [12] was used to set the three SVF gains such that the closed loop system had poles at $(s+250 \pm 4.33 i)$ and $(s+500)$. This produced very high feedback gains which would cause stability issues in real time application. 
Therefore they were scaled down and then empirically tuned slightly to allow for the non-linear nature of the leakage which was not modelled. This produced the tracking results seen in Figure 10 and 11.

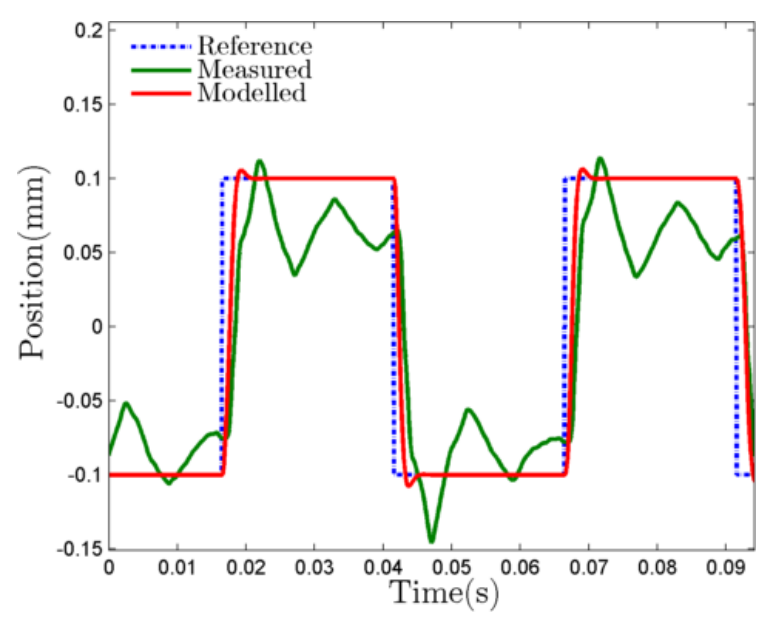

Figure 10: Comparison of valves actual and simulated response to SVF control at $20 \mathrm{~Hz}$

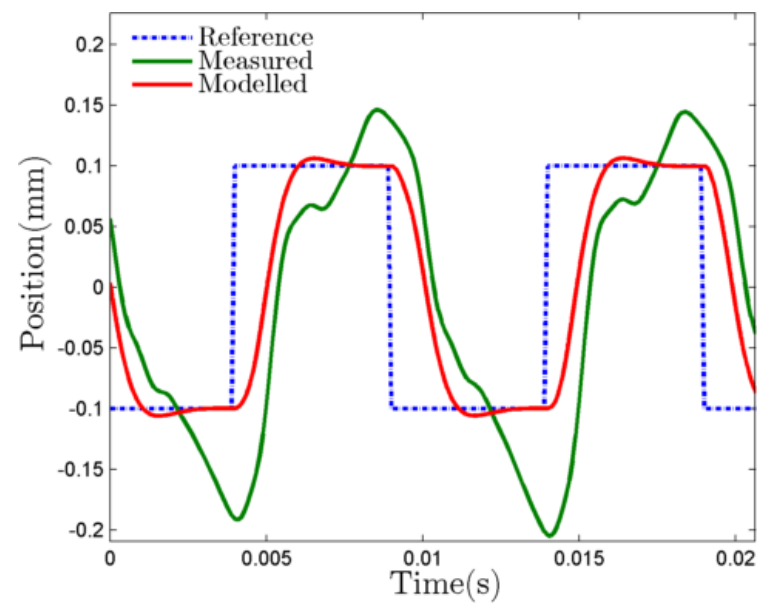

Figure 11: Comparison of valves actual and simulated response to SVF control at $100 \mathrm{~Hz}$

There is a large discrepancy between the simulated and obtained results. The lack of symmetry in the response of the valve at $100 \mathrm{~Hz}$ implies that the linear model is not able to account for the non-linear leakage. As with the comparison of the original model and experimental results the transient appears to be reasonably well modelled, but overshoot and beyond differ significantly. This is to be expected as it is during this period that the leakage will be most prevalent. Figure 11 makes two things clear. Firstly that a non-linear model of the valve is required and secondly that linear feedback control is not likely to prove sufficient to obtain a suitable response at $100 \mathrm{~Hz}$.

\section{Iterative Learning Control}

An Iterative Learning Controller was therefore used as a feedforward controller. Iterative Learning Control (ILC) first found widespread acceptance in 1984 with the publishing of a seminal paper by Arimoto et al [13]. Since then it has become one of the more popular forms of adaptive control due to its inherent simplicity. Consider a linear time invariable (LTI), single input single output (SISO) system.

$$
\begin{aligned}
& y_{k}=G(s) u_{k} \\
& e_{k}=y_{d}-y_{k}
\end{aligned}
$$

Where $y_{d}$ is the desired output, ILC works by modifying the input from the last iteration by some function of the error.

$$
\begin{aligned}
u_{k+1} & =u_{k}+L(s) e_{k} \\
& =u_{k}+L(s)\left(y_{d}-G(s) u_{k}\right) \\
y_{k+1} & =y_{k}+G(s) L(s)\left(y_{d}-y_{k}\right)
\end{aligned}
$$

Where $L(s)$ is the learning function. The block diagram for a simple ILC circuit is shown in Figure 12.

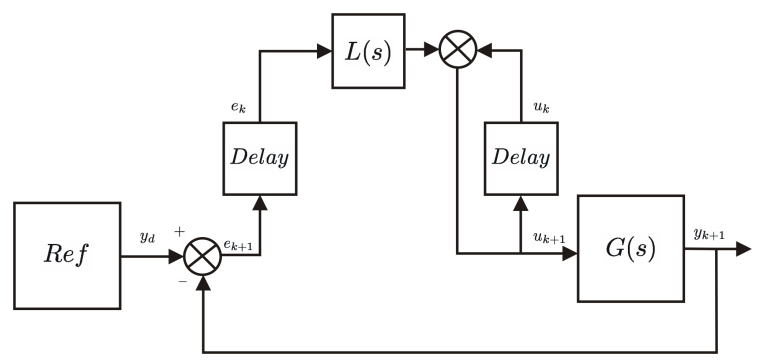

Figure 12: ILC Block Diagram

The success or failure of the controller is dependent only on the design of $L(s)$. It is apparent that the ideal learning function is a perfect inversion of the plant, as this would converge to a perfect solution after one iteration:

$$
\begin{aligned}
L(s) & =G(s)^{-1} \\
\therefore G(s) L(s) & =1 \\
y_{k+1} & =y_{k}+G(s) L(s)\left(y_{d}-y_{k}\right) \\
& =y_{k}+y_{d}-y_{k} \\
& =y_{d}
\end{aligned}
$$

Effectively ILC works by passing the error from the current iteration through an inverse model of the plant, then removing this from the input signal of the current iteration. The resulting signal is then used as the input for the next iteration. This means that if the inverse model were perfect then after a single iteration it would provide perfect tracking. It is however not possible to obtain a perfect inverse as no real plant is perfectly time invariant. It is also not possible to directly invert a non-minimum phase model, or to invert most non-linear models. However if the inverse is sufficiently close to the 'perfect' inverse it can be proved that the ILC will monotonically converge to an ideal solution [14] [15]. 
Classically the inverse model is derived by, either, modelling or identifying the system and then inverting the result, which can cause result in non-realisable or unstable inversions. Consequently the inverse models require the addition of fast poles or other modifications, reducing their accuracy. This also limits the inverse model to being linear in general, as most nonlinear models cannot be inverted. In order to avoid this extra step, and the extra inaccuracies it produces, it was proposed that instead an inverse model should be identified. This was achieved by using the high bandwidth position signal as the input for identification purposes and the input to the SVF loop as the output. This means that non-linear models can be used, though only with a modification to the classic ILC system. To ensure correctness it is necessary to pass the feedback signal through the inverse model and then subtract from the inverted desired signal to find the correction, rather than subtracting then passing through the model. The modification to the classic ILC block diagram can be seen in Figure 13 below.

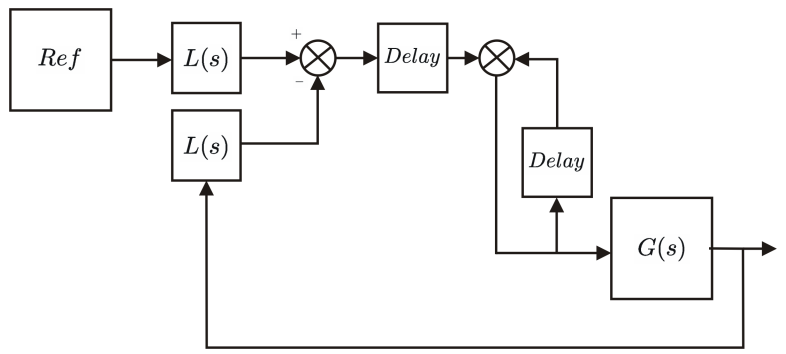

Figure 13: Block diagram for ILC with non-linear inverse model

The data used to identify the inverse model was a sine sweep between $10 \mathrm{~Hz}$ and $200 \mathrm{~Hz}$. This was to ensure that there would be sufficient data around the critical $100 \mathrm{~Hz}$ area without attempting to model higher order effects. The experimental data used can be seen below in Figure 14.

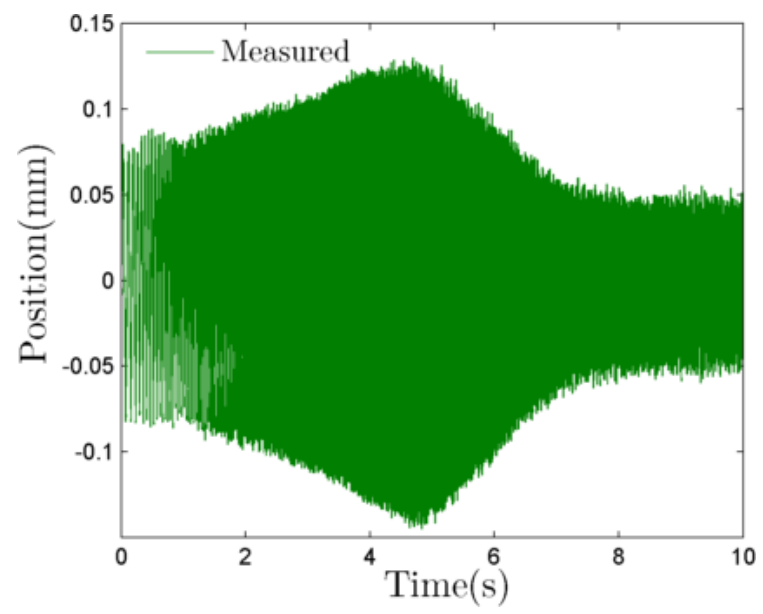

Figure 14: Valves response to a $0.1 \mathrm{~mm} 10-200 \mathrm{~Hz}$ sweep under SVF control

In order to account for the non-symmetrical leakage in the second stage of the valve a Hammerstein-Wiener model [16] was identified. This models input and output non-linearities and uses a linear model for the transient response, an example can be seen in Figure 15.

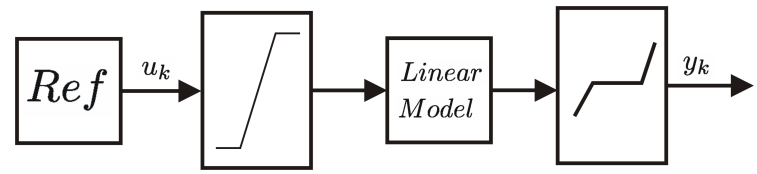

Figure 15: Example Hammerstein-Wiener model

The input and output non-linearities were modelled as piecewise linear functions. With the input function acting as a saturation block and the output block attempting to model the non-symmetrical valve leakage. The frequency response of the linear model can be seen in Figure 16.
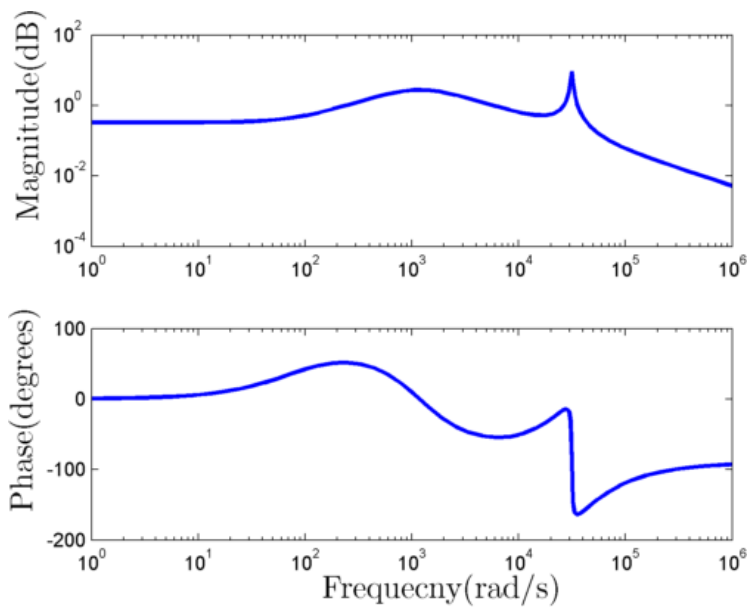

Figure 16: Frequency response of linear inverse model

For validation purposes the model was run on the position signal from a $100 \mathrm{~Hz}$ square wave experiment, Figure 17 shows that the model was effective at recreating the input square wave but with a pure time delay of $1 \mathrm{~ms}$. This delay can be accounted for in the ILC scheme by reducing the amount of delay on the corrective arm (before $L(s)$ ). Therefore this offset will not present under ILC.

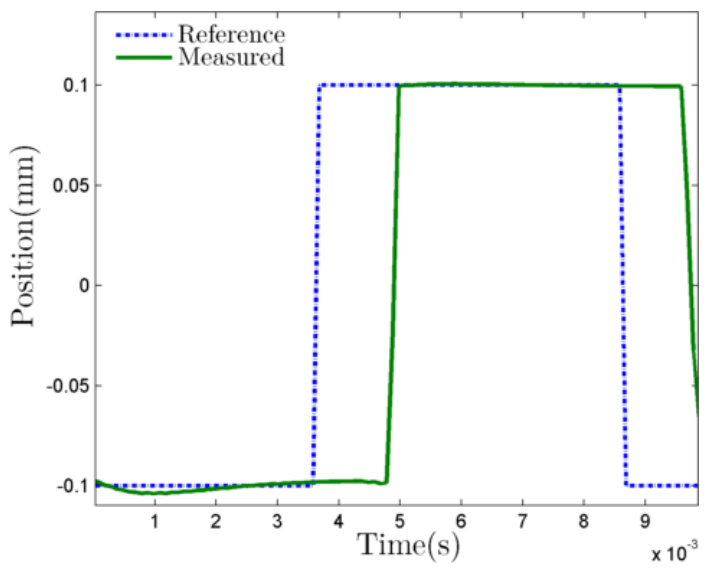

Figure 17: Validation of Inverse Model 
This inverse model was then used in the non-linear ILC scheme depicted in Figure 13. The convergence of the solution can be seen in Figure 18. A single cycle taken post convergence can also be seen in Figure 19. The input signal which generated this result is compared to the SVF input signal in Figure 20.

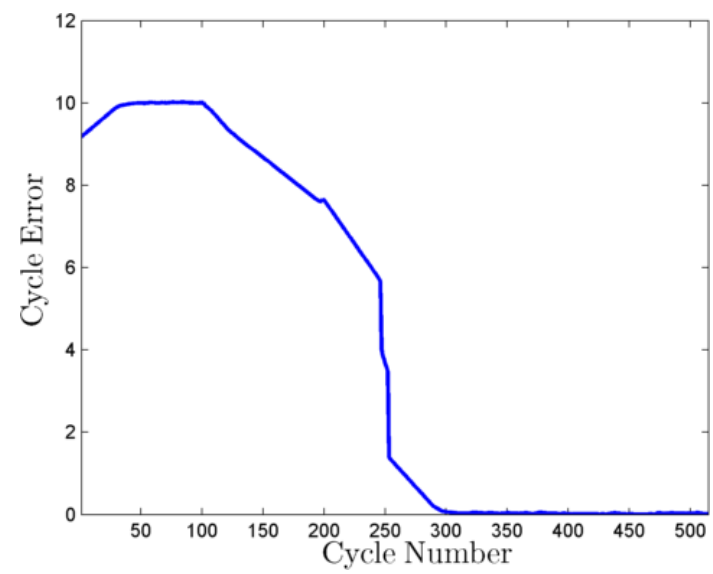

Figure 18: Smoothed cycle error of non-linear ILC

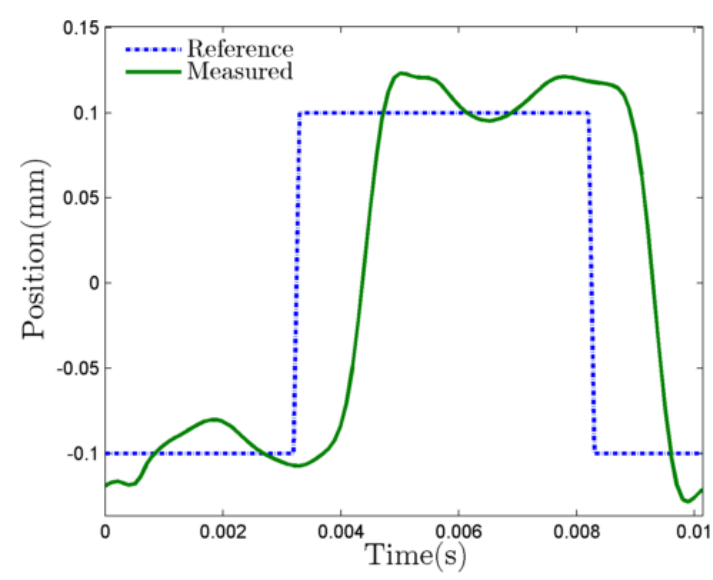

Figure 19: Valve response to ILC at $100 \mathrm{~Hz}$

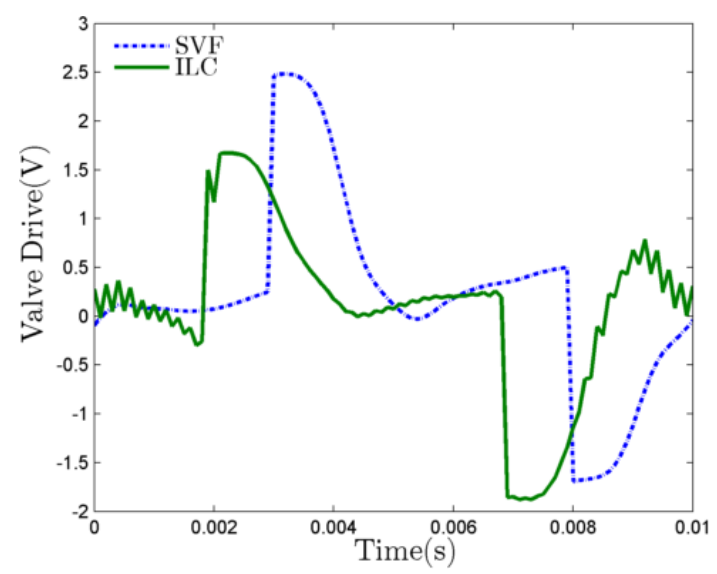

Figure 20: Comparison of input under ILC and SVF control
The solution takes around 300 cycles to converge, this is unacceptable for use in a SRHT, as the required response could be changed every second or less. The inclusion of the ILC dropped the rise time from $0.65 \mathrm{~ms}$ to $0.58 \mathrm{~ms}$. This is a small increase but the larger benefit can be seen in the difference of response post rise. Comparing the input from the SVF and ILC tests it can be seen that the ILC doesn't drive the pilot stage as hard as the SVF, meaning that it produces a smaller overshoot. However the ILC has a faster rise time than the SVF system. This is believed to be the result of not having to move from a stationary position and thus not needing to overcome the static friction in the valve. There is also a preemptive downturn at $2 \mathrm{~ms}$ that assists with reducing the overshoot. The sharp oscillations in the ILC response should be noted however.

The over long convergence time is the result of a low learning gain (only $1 \%$ of the calculated correction was applied each cycle) that was required to ensure stability in the basic ILC scheme. In order to allow for higher learning gains it is neccessary to build a more robust correction arm that is able to stop learning, once a sufficent response has been obtained. This will stop it from continuing to increase the initial transient once saturation has been reached. A low pass filter could also be added to stop the model trying to correct high frequency errors above the range of the valves response, such as can be seen in the input signal above (Figure 20).

\section{Conclusion}

The field of digital hydraulics is set to be pivotal in the future development of power transmission. It has the ability to compete with the on going advance of electrical drives by significantly improving the efficiency of hydraulic drives. Currently, however, the benefits of digital hydraulics have been realised on paper more than in reality. A SRHT is now closer to becoming a reality as it has been shown that it is possible to switch a high flow rate digital hydraulic valve at the $100 \mathrm{~Hz}$ required to make the concept viable.

Such a frequency was achieved by using a combination of SVF control and an ILC in the feed-forward path. Full state control by using a series of complementary filters to combine data from the position sensor and accelerometer to give high bandwidth position, velocity and acceleration measurements. SVF was sufficient to follow a $20 \mathrm{~Hz}$ square wave but suffered from significant undershoot. Therefore it was determined to use a feedforward controller to compliment the SVF. An inverse model ILC was used, with this inverse model being identified from empirical data using a non-linear Hammerstein-Wiener framework. This required a slight modification to the standard ILC circuit but proved effective up to $100 \mathrm{~Hz}$

Since these results were recorded the next design iteration of the valve has been developed to solve the non-symmetric leakage in the second stage. The Hammerstein-Wiener inverse model has also been exchanged for an auto-regressive neural network. This allows for a recursive inverse model that is able to adjust to a wide range of frequencies and pulse widths. Both of these changes are yet to be tested experi- 
mentally but it is believed that they will improve the dynamic response further.

\section{Acknowledgements}

This work is supported by UK Engineering and Physical Sciences Research Council (EPSRC EP/H024190/1), together with Instron, JCB and Parker Hannifin.

\section{Abbreviations}

\begin{tabular}{ll}
\hline Abbreviations & Definition \\
\hline SRHT & $\begin{array}{l}\text { Switched Reactance Hydraulic Trans- } \\
\text { former }\end{array}$ \\
PWM & Pulse Width Modulation \\
PID & Proportional Differential \& Integral \\
SVF & State Variable Feedback \\
SISO & Single Input Single Output \\
LTI & Linear Time Invariable \\
ILC & Iterative Learning Control \\
\hline
\end{tabular}

\section{References}

[1] M. Huova and A. Laamanen. Control of three-chamber cylinder with digital valve system. In Proceedings of The Second Workshop on Digital Fluid Power, Tampere, Finland, 2009.

[2] E. Bishop. Linearization of quantized digital hydraulic transformer output. In Proceedings of The Third Workshop on Digital Fluid Power, Tampere, Finland, 2010.

[3] I. Hyöty. Commercial high flow on/off-valves for digital hydraulics. In Proceedings of The Fifth Workshop on Digital Fluid Power, Tampere, Finland, 2012.

[4] J. P. Uusitalo, V. Ahola, L. Söderlund, M. Linjama, M. Juhola, and L. Kettunen. Novel bistable hammer valve for digital hydraulics. In Proceedings of The Second Workshop on Digital Fluid Power, Tampere, Finland, 2009.

[5] M. Karvonen, M. Ketonen, M. Linjama, and V. Puumala. Recent advancements in miniture valve development. In Proceedings of The Fourth Workshop on Digital Fluid Power, Tampere, Finland, 2011.

[6] H.C. Tu, M.B. Rannow, M. Wang, P. Y. Li, and T.R. Chase. Modeling and validation of a high speed rotaryt pwm on/off valve. In Proceedings of the ASME Dynamic System and Control Conference, 2009.

[7] M. Ketonen. Impementation of a digital hydraulic valve system with bosch rexroth sec valves. In Proceedings of The Fifth Workshop on Digital Fluid Power, Tampere, Finland, 2012.

[8] D.N Johnston. A switched inertance device for efficent control of pressure and flow. In Proceedings of the Bath/ASME conference of Fluid Power and Motion Control, Hollywood, 2009.
[9] S. Kudzma, D.N. Johnston, A.R. Plummer, and N.P. Sell. A high flow fast switching valve for digital hydraulic systems. In The Fifth Workshop on Digital Fluid Power, Tampere, 2012.

[10] A.R. Plummer. Optimal complementary filters and their application in motion measurement. Journal of syst. \& contr. engineering, 220(6), 2006.

[11] K. Ogata. Modern Control Engineering, chapter 9, pages 686-692. Pearson, 2010.

[12] K. Dutton, S. Thompson, and B. Barraclough. The Art of Control Engineering, chapter 5. State Variable Feedback. Addison-Wesley, 1998.

[13] S. Arimoto, S. Kawamura, and F. Miyazaki. Bettering operation of robots by learning. Journal of Robotic Systems, 2:123-140, 1984.

[14] K. L. Moore. An observation on monotonic convergence in discrete-time -p-type iterative learning control. In Proc.IEEE Int. Symp. Intell. Contr., Mexico City, 2001.

[15] N. Amman, D. H. Owens, and E. Rogers. Iterative learning control for discrete time systems with exponential rates of convergence. In IEE Proc Cntr. Thr. \& Applicat., 1996.

[16] A. H Falkner. 1988. International Journal of Control, 48(1):385-396, 1988 